\title{
ANÁLISE DE AGRUPAMENTO DE SINAIS OBTIDOS POR SIMULAÇÃO NUMÉRICA DO ENSAIO ULTRASSÔNICO PARA RECONHECIMENTO DE DEFEITOS DE SOLDAGEM *
}

\author{
Raphaella Hermont Fonseca Murta ${ }^{1}$ \\ Victor Oliveira Santos ${ }^{2}$ \\ Flávison de Almada Vieira ${ }^{3}$ \\ Paulo Jarbas Camurça ${ }^{4}$ \\ Elineudo Pinho de Moura ${ }^{5}$
}

Resumo

O ensaio não destrutivo por ultrassom é amplamente utilizado para detectar e dimensionar descontinuidades no interior do material. A técnica TOFD (Time Of Flight Diffraction [do inglês]) foi a técnica ultrassônica utilizada. Entretanto, a classificação do tipo de descontinuidade, a partir dos sinais ultrassônicos obtidos durante uma inspeção requer um profissional altamente capacitado. Esta tarefa pode ser realizada por algoritmos de reconhecimento de padrões, capazes de processar rapidamente uma grande quantidade de dados. Nesse trabalho, foram simulados três tipos de descontinuidades em juntas soldadas: falta de penetração, poro e trinca, utilizando-se o Método dos Volumes Finitos (MVF). Em seguida, foi aplicado o método de reconhecimento de padrões $\mathrm{K}$-vizinhos mais próximos (K-NN - K-nearest neighbors [do inglês]), para verificar como esses dados são separados. Esse trabalho é um passo inicial para o emprego de simulação numérica do ensaio ultrassônico para produzir sinais simulados e utilizá-los no treinamento de ferramentas de reconhecimento de padrão, a serem futuramente testados com sinais ultrassônicos experimentais. O K-NN classificou bem os sinais simulados. Conseguindo uma taxa média de acerto de aproximadamente $84 \%$.

Palavras-chave:Ultrassom; TOFD; MVF; K-NN.

\section{ANALYSIS OF GROUPING OF SIGNALS OBTAINED BY NUMERICAL SIMULATION OF ULTRASONIC TESTING FOR RECOGNITION OF WELDING DEFECT}

\section{Abstract}

The non-destructive ultrasound test is widely used to detect and size up discontinuities embedded a material. The TOFD (Time Of Flight Diffraction) was an ultrasound based technique used. However, the classification of the type of the discontinuity from the ultrasound signals acquired from an inspection requires a highly skilled professional. This task can be done by using patter recognition algorithms, which are able to quickly process a great amount of data. In this present work, three types of discontinuities (lack of penetration, porosity and crack) were simulated using the Finite Volume Method (FVM). After that, the method of pattern recognition K- nearest neighbor (K-NN) was used, to verify how these data are divided. This work is an initial step for the use of numerical simulation of the ultrasound simulation to generate simulated signs and use them in the training section of pattern recognition tools that which, in the future, can be tested with experimental ultrasound signals. Obtaining an average success rate of approximately $84 \%$.

Keywords: Ultrasound; TOFD; FVM; K-NN.

Engenharia Metalúrgica, Mestranda em Engenharia e Ciência dos Materiais, Bolsista de Mestrado, Departamento de Engenharia Metalúrgica e de Materiais, Universidade Federal do Ceará, Fortaleza, Ceará, Brasil. 
2 Engenharia Mecânica, Graduando em Engenharia Mecânica, Bolsista de Iniciação Cientifica, Departamento de Engenharia Metalúrgica e de Materiais, Universidade Federal do Ceará, Fortaleza, Ceará, Brasil.

3 Engenharia Mecânica, Graduado em Engenharia Mecânica, Universidade Federal do Ceará, Fortaleza, Ceará, Brasil.

4 Física, Mestrando em Engenharia e Ciência dos Materiais, Departamento de Engenharia Metalúrgica e de Materiais, Universidade Federal do Ceará, Fortaleza, Ceará, Brasil.

5 Engenharia Química, Doutor em Engenharia Metalúrgica e de Materiais, Professor Associado, Departamento de Engenharia Metalúrgica e de Materiais, Universidade Federal do Ceará, Fortaleza, Ceará, Brasil. 


\section{INTRODUÇÃO}

Ensaios não-destrutivos são usados para detectar imperfeições (descontinuidades) dentro de um material, sem impossibilitar seu uso posteriormente. Alguns destes testes conseguem detectar essas descontinuidades e determinar seu tamanho, como é o caso do ensaio por ultrassom, que usa ondas mecânicas de alta freqüência para detectá-las. Quando essa onda se propaga dentro do material, ela é difratada de volta para o sensor piezoelétrico, tornando possível determinar características do meio estudado, através da análise do sinal, chamado de A-scan, obtido pelo sensor receptor. Entre as técnicas ultrassônicas disponíveis, a técnica TOFD possui alta taxa de detecção de descontinuidades, tendo seu princípio baseado na difração causada na onda pelas extremidades superior e inferior da imperfeição. Contudo, a classificação do tipo de descontinuidade, a partir dos sinais ultrassônicos obtidos durante uma inspeção, requer um profissional altamente capacitado e bastante tempo. Essa tarefa pode ser realizada por algoritmos de aprendizagem de máquinas visando à classificação rápida de uma grande quantidade de dados. Os algoritmos de classificação podem ser treinados a reconhecerem padrões a partir de dados previamente conhecidos. Nesse trabalho, 0 algoritmo supervisionado K-NN foi o utilizado. Para que esse algoritmo classifique bem as descontinuidades em um material é preciso ter um grande banco de dados, com descontinuidades de diferentes tipos e tamanhos e uma forma fácil e barata de se obter isso é através da simulação. Foram simulados utilizando o Método dos Volumes Finitos três tipos de descontinuidades: falta de penetração, poro e trinca.

\section{MATERIAIS E MÉTODOS}

\subsection{Simulação Numérica}

A simulação numérica permite a obtenção de soluções de inúmeros problemas reais, para os quais a solução analítica é impraticável, imprecisa, cara ou que demanda muito tempo. Através dessa técnica é possível entender um problema real com a ajuda de operações elementares alcançando uma solução do problema.

Nesse trabalho, a simulação foi realizada utilizado o Método dos Volumes Finitos, que consiste em subdividir o domínio em volumes de controle e aplicar as leis de conservação, desta forma, o fluxo de entrada em um determinado volume será idêntico ao fluxo de saída. As equações aproximadas são obtidas a partir da integração das equações diferenciais em um volume de controle conhecido.

A modelagem de um meio bidimensional e isotrópico contendo descontinuidades típicas de juntas de soldas e a simulação numérica foi realizada utilizando-se a linguagem de programação FORTRAN.

\subsubsection{Propriedades do Material}

O material modelado é um aço carbono homogêneo e isotrópico. As suas características são apresentadas na Tabela 1. A geometria do sistema foi definida como sendo um retângulo de comprimento igual a 80 [mm] e largura igual a 19 [mm].

Tabela 1. Propriedades do material

Propriedades

Velocidade da onda longitudinal

Velocidade da onda transversal

Densidade
Valores Estabelecidos $5900[\mathrm{~m} / \mathrm{s}]$

$3200[\mathrm{~m} / \mathrm{s}]$

$7900\left[\mathrm{~kg} / \mathrm{m}^{3}\right]$




\subsubsection{Descontinuidades}

Os três tipos de descontinuidades (falta de penetração, poro e trinca) comumente encontradas em juntas de soldas foram modeladas. Para cada tipo, foram feitas 36 diferentes simulações, variando, dependendo do tipo, sua posição e suas dimensões, totalizando 108 sinais ultrassônicos. O objetivo da modificação dos parâmetros é criar um banco de dados com variabilidade de possibilidades que serão, posteriormente, utilizados para treinar o algoritmo de reconhecimento de padrões. Para a descontinuidade do tipo poro foram definidos dois tamanhos $(1,0$ $\mathrm{mm} \times 1,0 \mathrm{~mm}$ e $2,0 \mathrm{~mm} \times 2,0 \mathrm{~mm}$ ), e para cada tamanho a localização do poro no interior do material foi modificada aleatoriamente dezoito vezes, produzindo 36 sinais para essa classe de defeito. Para a descontinuidade do tipo trinca foram definidos quatro valores de comprimento, variando de $5 \mathrm{~mm}$ a $8 \mathrm{~mm}$ com incremento de $1 \mathrm{~mm}$. Estabeleceu-se que a largura da trinca corresponde a um décimo de seu comprimento, produzindo descontinuidades com dimensões 0,5 x 5,0 mm / 0,6 x 6,0 $\mathrm{mm} / 0,7 \times 7,0 \mathrm{~mm} / 0,8 \times 8,0 \mathrm{~mm}$. Para cada tamanho fez a posição da descontinuidade variar aleatoriamente nove vezes, gerando também 36 sinais. Para a descontinuidade do tipo falta de penetração foram utilizados seis valores de largura $(\mathrm{L}=1,0 / 2,0 / 3,0 / 4,0 / 5,0 / 6,0$ [mm]) e seis valores de altura $(\mathrm{a}=3,0 / 4,0$ / 5,0 / 6,0 / 7,0 / 8,0 [mm]), gerando um sinal para cada uma das 36 combinações. Vale ressaltar que esse tipo de descontinuidade aparece sempre na metade do comprimento da peça, e aberta à superfície inferior. A representação dos 3 tipos de descontinuidade é apresentada pela Figura 1.

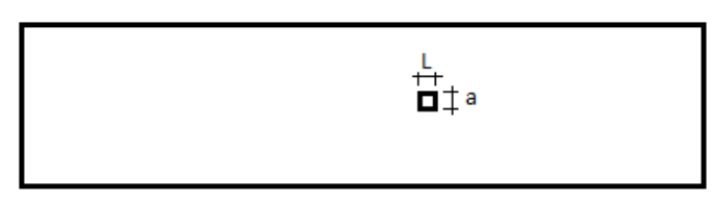

Poro

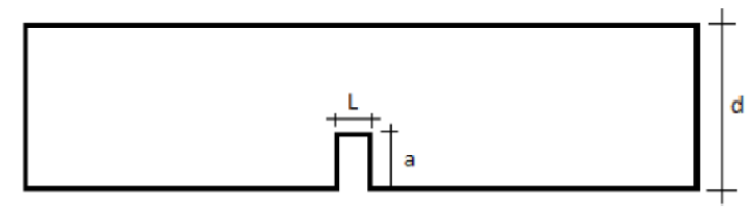

Falta de penetração

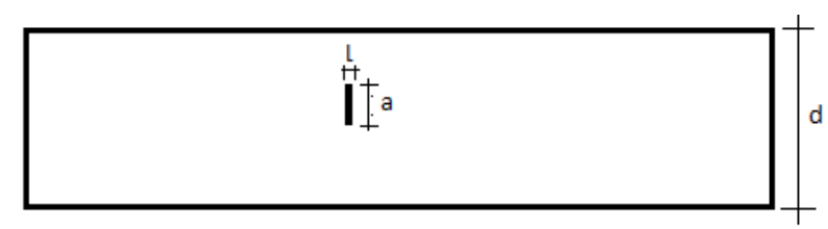

Trinca

Figura 1. Modelagem dos diferentes tipos de descontinuidade

\subsubsection{Transdutores}

Existe uma grande variedade de transdutores ultrassônicos no mercado. As principais diferenças encontradas entre eles são a freqüência de operação e o ângulo de incidência. Nos ensaios utilizando a técnica TOFD pode ser usado um transdutor com ângulo de incidência ajustável. Para simularmos esse transdutor utilizamos o princípio de Huygens, onde o acionamento seqüencial em diferentes tempos dos cristais de vibração do transdutor permite o direcionamento do feixe de onda. Um estudo extenso da influência de determinados parâmetros para a aquisição de um feixe ultrassônico ideal foi realizado [1]. Os parâmetros analisados foram: comprimento de cada cristal (a), espaçamento entre os cristais (d), 
quantidade de cristais $(N)$ e ângulo de incidência $(\theta)$, como pode ser observado na Figura 2.

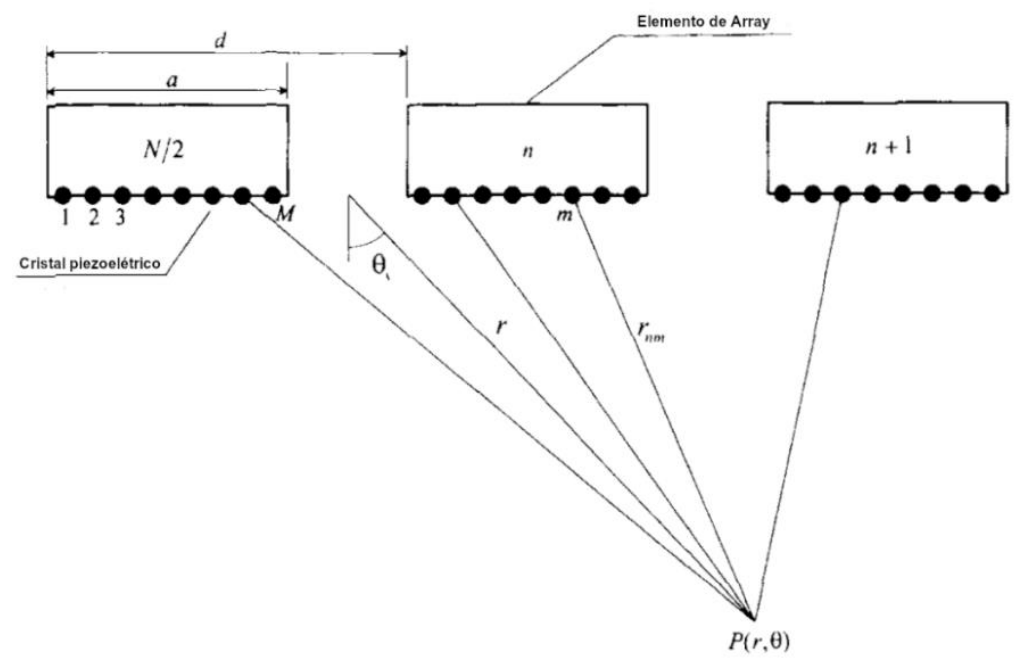

Figura 2. Representação dos parâmetros do transdutor fonte

A Tabela 2 mostra todos os valores utilizados para os parâmetros referentes aos transdutores na modelagem computacional.

Tabela 2. Valores referentes aos parâmetros do transdutor fonte

\begin{tabular}{cc}
\hline Descrição & Valor \\
\hline Ângulo de incidência $(\theta)$ & $60^{\circ}$ \\
\hline Quantidade de cristais $(\mathrm{n})$ & 8 \\
\hline Quantidade de pontos vibrantes $(\mathrm{N})$ & 8 \\
\hline Comprimento de onda $(\lambda)$ & $1,180 \mathrm{E}-3[\mathrm{~m}]$ \\
\hline Frequência da onda $(\mathrm{f})$ & $5,0 \mathrm{E} 6[\mathrm{~Hz}]$ \\
\hline Espaçamento entre cristais $(\mathrm{d})$ & $8,43 \mathrm{E}-5[\mathrm{~m}]$ \\
\hline Espaçamento entre elementos vibrantes do cristal & $5,9 \mathrm{E}-4[\mathrm{~m}]$ \\
\hline Tempo de intervalo de acionamento entre cristais & $6,93 \mathrm{E}-2[\mathrm{~m}]$ \\
\hline
\end{tabular}

\subsubsection{Equação da onda}

A equação do movimento em um meio bidimensional é dada pela quantidade de movimento, que pode ser expressa pela segunda lei de Newton[2], como:

$$
\rho \frac{\partial^{2} u_{i}}{\partial t^{2}}=F_{i}+\frac{\partial \tau_{i j}}{\partial x_{j}}
$$

onde $\boldsymbol{\rho}$ é a densidade; u é o vetor de deslocamento; t é o tempo; F é a força por unidade de volume e $\tau$ é a tensão. Neste trabalho, o meio simulado é elástico, podendo assim ser utilizado a lei de Hooke [3], dada pela Equação 2.

$$
\tau_{\mathrm{ij}}=\mathrm{C}_{\mathrm{ijkl}} \varepsilon_{\mathrm{kl}}
$$

onde $\varepsilon_{\boldsymbol{k} l}$ e $\boldsymbol{C}_{\boldsymbol{i} \boldsymbol{j} \boldsymbol{k} \boldsymbol{l}}$ são, respectivamente, a deformação do material (Equação 3) e o módulo elástico (Equação 4). 


$$
\begin{array}{r}
\varepsilon_{\mathrm{kl}}=\frac{1}{2}\left(\frac{\partial u_{\mathrm{k}}}{\partial \mathrm{x}_{\mathrm{l}}}+\frac{\partial \mathrm{u}_{\mathrm{l}}}{\partial \mathrm{x}_{\mathrm{k}}}\right) \\
\mathrm{C}_{\mathrm{ijkl}}=\lambda \delta_{\mathrm{ij}} \delta_{\mathrm{kl}}+\mu\left(\delta_{\mathrm{ik}} \delta_{\mathrm{jl}}+\delta_{\mathrm{il}} \delta_{\mathrm{jk}}\right)
\end{array}
$$

$\lambda, \boldsymbol{\mu}$ e $\boldsymbol{\delta}$ são, respectivamente, o parâmetro de Lamé, o módulo de cisalhamento e o delta de Kronecker. Substituindo as Equações 2, 3 e 4 na Equação 1 e a desenvolvendo, tendo em vista que o material simulado é isotrópico, obtêm-se as equações do movimento ao longo de x e y (Equações 5 e 6).

$$
\begin{aligned}
& \rho \frac{\partial^{2} u_{x}}{\partial t^{2}}=F_{x}+\frac{\partial}{\partial x}\left(C_{1111} \frac{\partial u_{x}}{\partial x}\right)+\frac{\partial}{\partial x}\left(C_{1122} \frac{\partial u_{y}}{\partial y}\right)+\frac{\partial}{\partial y}\left(C_{1221} \frac{\partial u_{x}}{\partial y}\right)+\frac{\partial}{\partial y}\left(C_{1111} \frac{\partial u_{y}}{\partial x}\right) \\
& \rho \frac{\partial^{2} u_{y}}{\partial t^{2}}=F_{y}+\frac{\partial}{\partial x}\left(C_{1221} \frac{\partial u_{x}}{\partial y}\right)+\frac{\partial}{\partial x}\left(C_{1221} \frac{\partial u_{y}}{\partial x}\right)+\frac{\partial}{\partial y}\left(C_{1122} \frac{\partial u_{x}}{\partial x}\right)+\frac{\partial}{\partial y}\left(C_{1111} \frac{\partial u_{y}}{\partial y}\right)
\end{aligned}
$$

\subsubsection{Condições de Contorno}

Para que a simulação fique o mais próximo possível do ensaio ultrassônico real, é necessário um conjunto de restrições adicionais nos limites da peça, chamadas de condições de contornos. Elas são utilizadas com a intenção de limitar a porção do espaço, sobre o qual a solução da equação da onda é calculada, deixando assim, a solução mais próxima daquela obtida, quando não há existência de limitações artificiais. Nesse trabalho, foram utilizadas as condições de superfície livre, bordas não reflexivas, camadas de amortecimento e deslocamento nulo, que estão sendo ilustradas na Figura 3 e brevemente explicadas nas subseções 2.2.3.1, até 2.2.3.4.

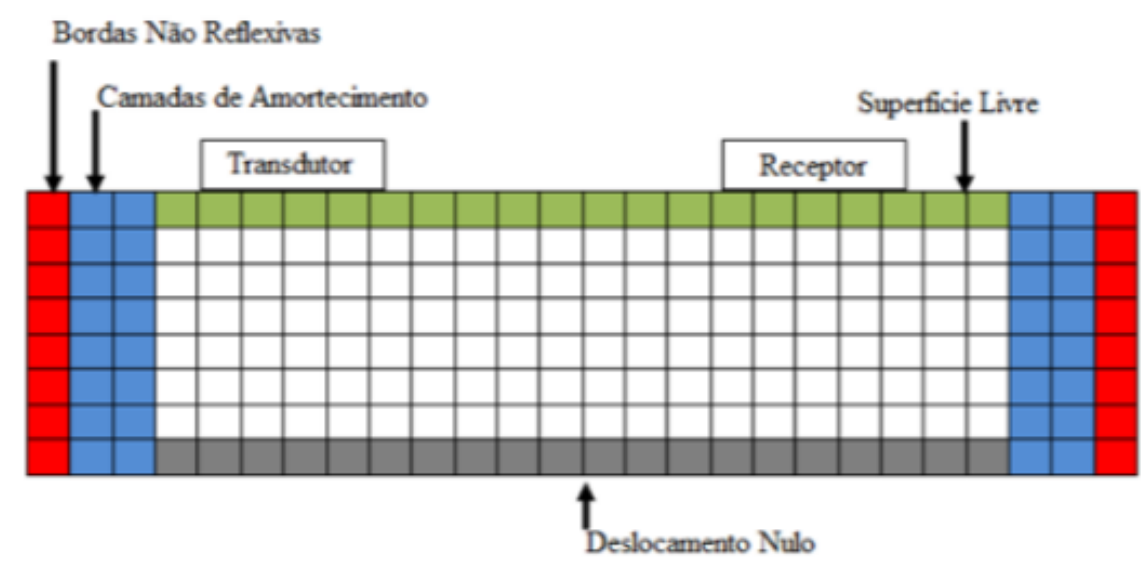

Figura 3. Representação esquemática das condições de contorno do material.

\subsubsection{Superfície livre}

A condição de contorno de superfície livre é utilizada nos problemas que ocorrem em um curto intervalo de tempo, fazendo com que, o efeito da superfície não seja importante para a simulação. A condição de contorno foi aplicada na superfície superior da peça, onde as tensões normais são nulas. As Equações 7 e 8 representam o efeito da superfície livre em um material [4].

$$
\begin{array}{r}
\frac{\partial u_{x}}{\partial y}+\frac{\partial u_{y}}{\partial x}=0 \\
\mathbf{v}_{p}^{2}\left(\frac{\partial u_{y}}{\partial y}\right)+\left(v_{p}^{2}-2 v_{s}^{2}\right) \frac{\partial u_{x}}{\partial x}=0
\end{array}
$$




\subsubsection{Bordas não reflexivas}

$\mathrm{Na}$ simulação, quando as ondas ultrassônicas atingem as bordas laterais, elas sofrem variações abruptas, devido as reflexões ocasionadas pela diferença de impedância entre metal e o ar [5]. A fim de se evitar essa variação, faz-se necessário a aplicação da condição de contorno de bordas não reflexiva nas extremidades laterais esquerda e direita da peça. Essa condição de contorno assume que as bordas laterais estão suficientemente distantes, de modo que, podemos considerar as frentes de onda planas nesta região. A condição é dada pela Equação 9.

$$
\frac{1}{v} \frac{\partial^{2} u}{\partial t^{2}}-\frac{\partial^{2} u}{\partial x^{2}}-\frac{\partial^{2} u}{\partial y^{2}}=0
$$

\subsubsection{Camadas de amortecimentos}

As camadas de amortecimentos foram aplicadas nas regiões da malha próximas das bordas laterais da peça, como está sendo ilustrado na Figura 3. O objetivo dessa condição de contorno é evitar que a onda ultrassônica tenha energia e se propague no material após incidir nas extremidades, direita e esquerda, da peça. A idéia é reduzir gradativamente as amplitudes da onda, através da multiplicação do campo de deslocamento, por um fator de absorção exponencial, de modo que ao atingir as extremidades da peça a amplitude da onda seja nula. O fator de amortecimento é dado pela Equação 10, para a extremidade esquerda do modelo e pela Equação 11, para a extremidade direita do modelo [6].

$$
\begin{array}{r}
W(k)=\exp \left(-\left(f_{a t}\left(N_{a}-k\right)\right)^{2}\right) \\
W(k)=\exp \left(-\left(f_{a t}\left(k-\left(N_{x}-N_{a}\right)\right)\right)^{2}\right)
\end{array}
$$

onde $\mathrm{W}(\mathrm{k})$ é o fator de atenuação; fat é o fator de amortecimento; $\mathrm{N}_{\mathrm{a}}$ é o número de volumes utilizados para atenuar a onda; $k$ é o índice do volume da malha e $N_{x}$ é o número de volumes na direção $x$.

\subsubsection{Deslocamento nulo}

Devido à grande diferença de impedância entre metal e o ar, a onda, ao chegar na superfície inferior, tem a sua fase invertida e é quase completamente refletida. Para se evitar esforços computacionais, ao invés de acrescentarmos ao problema o ambiente externo, aplica-se a condição de contorno deslocamento nulo. A Equação 12 garante a reflexão total na superfície inferior do material [7].

$$
u_{x}=u_{y}=0
$$

\subsubsection{Tamanho da malha}

O tamanho da malha é uma das variáveis mais importantes na solução de problemas utilizando os métodos numéricos. Quanto mais refinada ela é, menores são os erros de truncamento associados ao problema, entretanto, maior será os esforços computacionais necessários à obtenção da solução numérica.

Para a escolha do comprimento de malha adequado foi realizado um estudo de refino de malha. Observa-se que, o tamanho de malha tem que ser proporcional à 
distância entre os cristais piezoelétricos que é de, para que o cristal esteja sobre o nó da malha.

Foram criadas malhas cartesianas estruturadas com comprimento de $\lambda / 112, \lambda / 126$, $\lambda / 140$ e $N / 154$, todas as variáveis do sistema foram mantidas, sendo portanto , 0 tamanho da malha a única distinção entre elas. A malha escolhida é aquela que quando refinada a solução não muda. Para essa simulação, $N 140$ foi o comprimento de malha utilizado.

\subsection{Pré-processamento dos sinais}

Após a aquisição dos sinais A-scan, modificações ainda podem ser feitas nos dados antes de serem utilizados no método de reconhecimento de padrões. O objetivo das modificações é identificar qual a forma de dados que irá fornecer os melhores resultados em termos de classificação. Um estudo anterior conclui que há melhoras na classificação utilizando-se a envoltória do sinal A-Scan [8]. Tal envoltória é obtida utilizando-se a aplicação do filtro Savitzky-Golay. Uma outra possibilidade, é a normalização do sinal A-scan, utilizando-se média igual a zero e desvio padrão igual a um. Dessa forma, três diferentes conjuntos de dados foram utilizados: sinais simulados, sinais normalizados e envoltórias dos sinais simulados.

\subsection{Reconhecimento de Padrões}

O reconhecimento de padrões é uma área da ciência que tem por objetivo a classificação/agrupamento de objetos em um número de categorias, através da utilização de algoritmos de classificação, que observam padrões a partir de um conjunto de propriedades ou características.

Existem dois tipos de algoritmos de classificação: o supervisionado e o não supervisionado. No classificador supervisionado, é apresentado ao computador exemplos de inputs e outputs desejados e o algoritmo tem como objetivo aprender uma regra geral, que relaciona, corretamente, os inputs aos outputs. Já no classificador não supervisionado, nenhum tipo de informação é dada ao algoritmo de aprendizado, deixando-o sozinho para encontrar a melhor maneira de agrupar os seus inputs. Neste trabalho, foi utilizado o k-NN, que é um algoritmo de aprendizado supervisionado.

\subsection{1 k-NN}

O método k-NN faz parte do conjunto de classificadores não-paramétricos para estimação de densidade, utilizando a regra de Bayes [9]. Inicialmente, aproximadamente $70 \%$ dos sinais são utilizados para treinamento do classificador e o restante é utilizado para teste. O procedimento do método é basicamente uma análise das distâncias euclidianas entre as variáveis referentes a cada sinal. Logo, num dado de teste, são calculadas as distâncias entre ele e os dados de treinamento. Verifica-se a quais classes pertencem os $\mathrm{k}$ dados de treinamento mais próximos e a classificação é feita associando-se o dado de teste à classe que for predominante entre esses dados. Por exemplo, sendo $k$ igual a um, o caso mais simples, a classe atribuída ao dado de teste será igual ao dado de treinamento mais próximo. Não existe uma regra geral para o valor ideal de $k$, logo testes são necessários. Realizou-se testes variando o parâmetro k de 1 a 4 e as melhores classificações foram consideradas. Os resultados são apresentados na forma de 
uma matriz chamada de matriz de confusão. Nela, cada coluna representa o tipo de descontinuidade que foi analisada e, cada linha, como ela foi classificada. Calculando-se a média da diagonal principal, obtêm-se a taxa média de acerto na classificação dos dados de teste. Como a separação dos dados é aleatória dependendo de quais dados são sorteados para treino e para teste a taxa média de acerto pode variar. Para que isso não influencie no resultado, o algoritmo foi repetido 100 vezes e uma matriz de confusão com a média das 100 anteriores foi obtida.

\section{RESULTADOS E DISCUSSÃO}

\subsection{Campo de deslocamento e A-scan}

O ensaio ultrassônico foi modelado em um aço comum contendo um poro, uma trinca ou uma falta de penetração. Cada simulação produziu um campo de deslocamento e um A-scan que fornece, respectivamente, informações qualitativas e quantitativas a simulação.

O campo de deslocamento é a representação da propagação da onda ao longo do material. O A-scan é um gráfico de amplitude pelo tempo que é gerado através dos sinais capturados pelo transdutor receptor. Através do tempo do percurso de onda, profundidade do material e ângulo de incidência da onda é possível dimensionar, localizar e classificar uma descontinuidade. Na Figura 4 e 5, tem-se, respectivamente, o campo de deslocamento no instante que a onda encontra 0 defeito para uma peça contendo uma descontinuidade do tipo falta de penetração, com altura de 3 [mm] e comprimento de 1 [mm] e o A-scan gerado por essa descontinuidade.

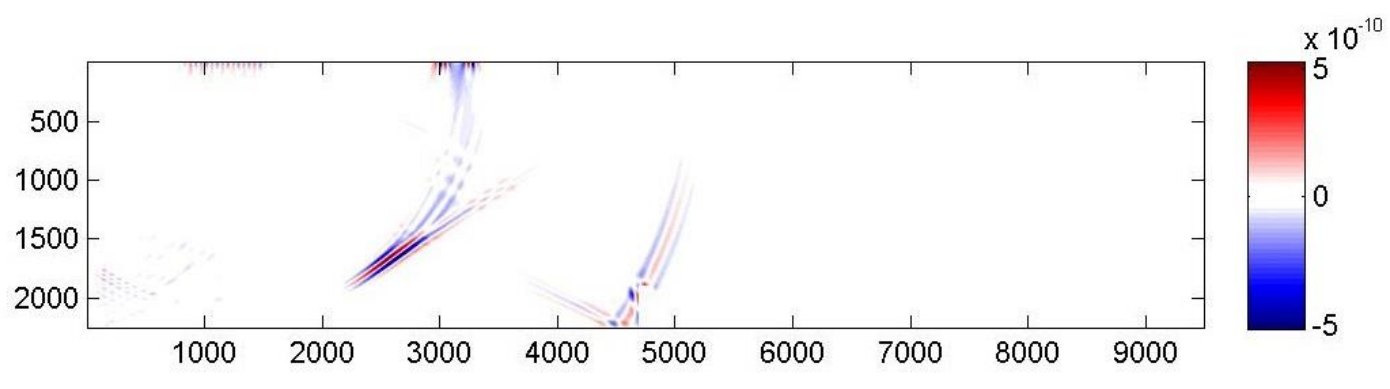

Figura 4. Campo de deslocamento gerado no instante em que a onda encontra uma descontinuidade do tipo falta de penetração $(\mathrm{h}=3[\mathrm{~mm}]$ e $\mathrm{l}=1[\mathrm{~mm}])$

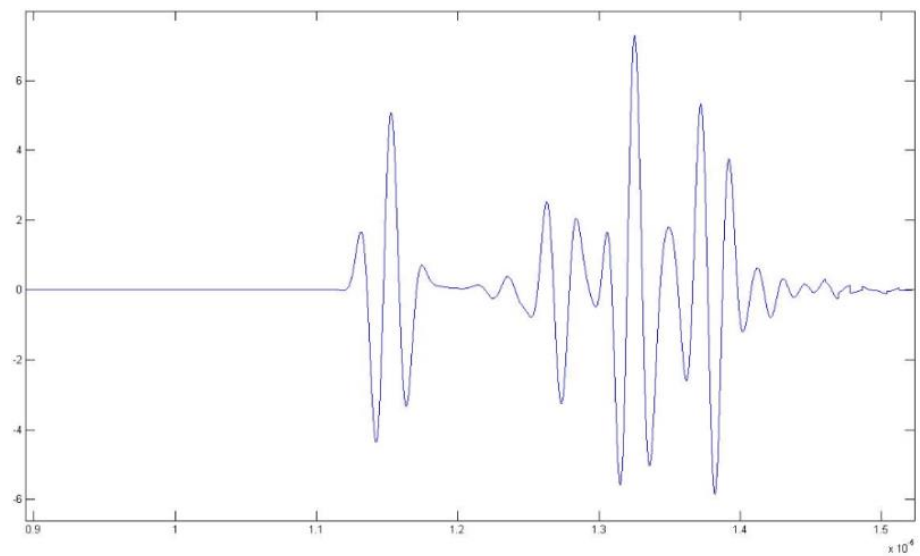

Figura 5. A-scan gerado por um modelo contendo uma descontinuidade do tipo falta de penetração $(\mathrm{h}=3[\mathrm{~mm}]$ e $\mathrm{l}=1[\mathrm{~mm}])$ 
Produziu-se 36 sinais de A-scan para cada tipo de descontinuidade, totalizando 108 sinais. Esses são os sinais que serão tratados/processados e, posteriormente, utilizados no algoritmo de reconhecimento de padrões.

\subsection{Análise de agrupamento dos sinais ultrassônicos simulados}

Os sinais ultrassônicos simulados foram processados e a melhor classificação foi obtida quando $\mathrm{k}=1$, a matriz de confusão é mostrada na Tabela 3. Observa-se que 99.85\% dos dados associados à falta de penetração foram corretamente classificados como falta de penetração. Aproximadamente $73 \%$ dos dados pertencentes a classe poro foram reconhecidos como poro, e que cerca de $79 \%$ dos dados referentes às trincas foram corretamente identificados como trincas. A taxa média de acerto na classificação foi de $83.86 \%$.

Tabela 3. Matriz de confusão obtida pelo emprego do k-NN aos sinais ultrassônicos simulados

$$
\text { Falta de Penetração Poro Trinca }
$$

\begin{tabular}{lccc}
\hline $\begin{array}{l}\text { Classificado como } \\
\text { Falta de Penetração }\end{array}$ & $99,85 \%$ & $8,57 \%$ & $8,14 \%$ \\
\hline $\begin{array}{l}\text { Classificado como } \\
\text { Poro }\end{array}$ & $0,15 \%$ & $72,58 \%$ & $12,71 \%$ \\
\hline $\begin{array}{l}\text { Classificado como } \\
\text { Trinca }\end{array}$ & $0 \%$ & $18,85 \%$ & $79,15 \%$ \\
\hline
\end{tabular}

\subsection{Análise de agrupamento dos sinais ultrassônicos simulados normalizados}

Os sinais ultrassônicos simulados normalizados apresentaram uma melhor classificação quando $k=1$, a matriz de confusão é mostrada na Tabela 4. Observa-se que a classificação dos sinais de falta penetração normalizados foi a mesma em comparação com os sem nenhum tratamento (99.85\%). Aproximadamente $78 \%$ dos dados pertencentes a classe poro foram identificados como poro, e que cerca de $71 \%$ dos dados referentes às trincas foram classificados como trincas. A taxa média de acerto passou de $83.86 \%$ para $82.76 \%$ com a normalização dos sinais.

Tabela 4. Matriz de confusão obtida pelo emprego do k-NN aos sinais ultrassônicos simulados normalizados

$$
\text { Falta de Penetração Poro Trinca }
$$

\begin{tabular}{lccc}
\hline $\begin{array}{l}\text { Classificado como } \\
\text { Falta de Penetração }\end{array}$ & $98,85 \%$ & $8,28 \%$ & $1,14 \%$ \\
\hline $\begin{array}{l}\text { Classificado como } \\
\text { Poro }\end{array}$ & $0 \%$ & $78,15 \%$ & $27,57 \%$ \\
\hline $\begin{array}{l}\text { Classificado como } \\
\text { Trinca }\end{array}$ & $1,15 \%$ & $13,57 \%$ & $71,29 \%$ \\
\end{tabular}




\subsection{Análise de agrupamento das envoltórias dos sinais ultrassônicos simulados}

A envoltória dos sinais ultrassônicos simulados também apresentou uma melhor classificação quando $k=1$. Os sinais de teste de falta de penetração e de trinca apresentaram classificações parecidas com as obtidas para os sinais sem nenhum tratamento e os normalizados $(99 \%$ de acerto para falta de penetração e $76 \%$ de acerto para trinca). Entretanto, houve uma piora significativa na classificação dos sinais do tipo poro, a porcentagem de acerto desse tipo de descontinuidade ficou em torno de $54 \%$. A matriz de confusão é apresentada na Tabela 5 . A taxa média de acerto na classificação foi de $76.48 \%$.

Percebe-se que a utilização da envoltória dos sinais ocasionou uma piora significativa na taxa média de acerto. Esse resultado parece ser incoerente com os obtidos no trabalho que inspirou o uso da envoltória dos sinais [8]. No entanto, o trabalho citado empregou classificadores não-lineares implementados por uma rede neural artificial, uma ferramenta mais complexa do que o K-NN. Trabalhos anteriores também não obtiveram resultados favoráveis ao uso da envoltória dos sinais na regra da classe mais próxima [10].

Tabela 5. Matriz de confusão obtida pelo emprego do k-NN a envoltória dos sinais ultrassônicos simulados

\begin{tabular}{lccc}
\hline & Falta de Penetração & Poro & Trinca \\
\hline $\begin{array}{l}\text { Classificado como } \\
\text { Falta de Penetração }\end{array}$ & $99 \%$ & $12,85 \%$ & $11,43 \%$ \\
\hline $\begin{array}{l}\text { Classificado como } \\
\text { Poro }\end{array}$ & $0,15 \%$ & $54,43 \%$ & $12,57 \%$ \\
\hline $\begin{array}{l}\text { Classificado como } \\
\text { Trinca }\end{array}$ & $0,85 \%$ & $32,72 \%$ & $76 \%$ \\
\hline
\end{tabular}

\section{CONCLUSÃO}

O k-NN classificou bem os sinais simulados. Alcançou-se uma taxa média de acerto de $84 \%$ para os sinais sem nenhum tratamento.

A falta de penetração foi a descontinuidade melhor classificada, isso se deu, possivelmente, pelo fato dessa descontinuidade sempre ocorrer no centro do cordão de solda e ter dimensões significativamente maiores do que as outras.

A classificação dos sinais normalizados e sem nenhum tratamento foi semelhante, já quando é aplicada a envoltória dos sinais no k-NN a taxa média de acerto diminuiu.

\section{Agradecimentos}

Ao CNPq pela bolsa PIBIC 148044/2016-6 do ano de 2016/2017.

A CAPES pelas bolsas de mestrado do de 2014/2015 e do ano de 2016/2017 concedidas. 


\section{REFERÊNCIAS}

$1 \mathrm{WOOH}$, S.-C.; SHI, Y. A simulation study of the beam steering characteristics for linear phased arrays. Journal of nondestructive evaluation, Springer, v. 18, n. 2, p. 39-57, 1999.

2 ROSE, J. L.Ultrasonic waves in solid media. [S.I.]: Cambridge university press, 2004

3 RYCHLEWSKI, J. On hooke $s$ law. Journal of Applied Mathematics and Mechanics.Elsevier, v. 48, n. 3, p. 303-314, 1984.

4 LANDAU, L. D.; LIFSHITZ, E. M. Course of Theoretical Physics Vol 7: Theory and Elasticity. [S.I.]: Pergamon press, 1959,p 96.

5 CATUNDA, C. E. G. Implementação da modelagem elástica para detecção de defeitos por ultra-som em juntas soldadas dissimilares. Universidade Federal do Rio de Janeiro (UFRJ), 2004.

6 CERJAN, C.; KOSLOFF, D.; KOSLOFF, R.; RESHEF, M. A nonreflecting boundary condition for discrete acoustic and elastic wave equations. Geophysics, Society of Exploration Geophysicists, v. 50, n. 4, p. 705-708, 1985

7 CAMURÇA, P. J. Simulação do ensaio ultrassônico pela técnica tofd utilizando o método dos volumes finitos. Universidade Federal do Ceará (UFC), 2014

8 MOURA, E. de; SIQUEIRA, M.; SILVA, R. da; REBELLO, J. Welding defect pattern recognition in tofd signals part 2. non-linear classifiers. Insight-Non-Destructive Testing and Condition Monitoring, The British Institute of Non-Destructive Testing, v. 47, n. 12, p.783-787, 2005

9 WEBB, A. R. Statistical pattern recognition. [S.I.]: John Wiley \& Sons, 2003.

10 MURTA, R. H. F. Análise de agrupamento de sinais obtidos por simulação do ensaio ultrassônico pela técnica TOFD para reconhecimento de defeitos de soldagem modelados. 2015. Monografia (Engenharia Metalúrgica), UFC (Universidade Federal do Ceará), Ceará, Brazil. 